\title{
Mini-laparotomy in advanced ovarian cancer
}

\author{
S. Sircar • G. J. Robson • J. A. Davis • J. K. Kennedy • \\ F. Alexander-Sefre
}

Received: 5 July 2011 / Accepted: 14 October 2011 /Published online: 18 November 2011

(C) Springer-Verlag 2011

\begin{abstract}
Optimal cytoreduction (OCR) remains the gold standard treatment of ovarian cancer. Current radiological imaging has limited sensitivity and specificity in prediction of achieving OCR prior to surgery. This prospective pilot study included 50 patients with advanced ovarian carcinoma. Prior to the main laparotomy, a mini-laparotomy_ just large enough to allow a hand in - was performed. A decision was then made as to whether achieving OCR is "possible", "not possible" or lastly "unsure". Formal laparotomy then followed. At the end of the formal laparotomy, cytoreduction was regarded as either "optimal" or "suboptimal" based on residual disease. Out of 45 cases where results were deemed suitable for analysis, 27 were regarded as "OCR possible", out of which OCR was achieved in 24 cases following full laparotomy. Ten were commented upon as "unsure" and only in three cases OCR was feasible. Eight were classed as "OCR not possible" and in none of these OCR was obtained. The only noted complication associated with minilaparotomy was bleeding in just three cases $(6 \%)$. The sensitivity, specificity, PPV and NPV of mini-laparotomy were $100 \%, 73 \%, 89 \%$ and $100 \%$, respectively. However, when "unsure", only in $30 \%$ OCR was achieved. We concluded that mini-laparotomy is a safe, simple and effective technique for predicting feasibility of
\end{abstract}

\footnotetext{
S. Sircar · G. J. Robson · J. A. Davis · J. K. Kennedy •

F. Alexander-Sefre

Department of Gynaecological Oncology,

Glasgow Royal Infirmary,

Glasgow G4 OSF Scotland, UK

F. Alexander-Sefre $(\bowtie)$

Ward 56B, Glasgow Royal Infirmary,

Castle Street,

Glasgow G4 0SF Scotland, UK

e-mail: f.alexander.sefre@ggc.scot.nhs.uk
}

OCR. This simple technique could obviate the need for full laparotomy in patients who may benefit from neo-adjuvant chemotherapy.

Keywords Mini-laparotomy · Optimal · Resectability · Advanced ovarian malignancy. Ovarian cancer

\section{Background}

Ovarian cancer is the most common gynaecological malignancy in the United Kingdom with nearly 7,000 reported cases per year [1]. The mainstay of treatment remains cytoreductive surgery followed by adjuvant chemotherapy. Most patients present with omental and peritoneal metastasis, hence making complete surgical resection a challenge [2]. Overall survival greatly depends on the possibility of resecting most of the diseased areas with little residual disease (optimal cytoreduction [OCR]). In early stages (I and II), when the disease is confined to the pelvis, the overall survival exceeds $70 \%$ whilst in advanced stages (III and IV) the 5-year survival is less than $40 \%$ [3]. In Scotland alone, there are over 600 reported cases per annum, of which over 400 succumb to the disease, hence an overall reported survival of $30 \%$ [4].

Achievement of OCR is the single most important factor for survival in patients with ovarian cancer [5]. Currently the preoperative selection of patients for primary surgery is mainly based on detailed imaging [6, 7]. The commonest imaging modality is computer tomography (CT) in most centres. CT accuracy varies greatly in assessment of resectability and prediction of OCR in advanced stage ovarian cancer [8]. Other preoperative assessment methods such as laparoscopy have not as yet been established in most centres. 
This pilot study aims to evaluate the role of minilaparotomy as an alternative or complementary (to CT imaging) means of determining tumour resectability and the chances of achieving OCR in advanced stage ovarian cancer.

\section{Methods}

A total of 50 consecutive patients with stage III/IV ovarian cancer were included in this prospective study. The study was conducted in the department of gynaecological oncology at Glasgow Royal Infirmary, over a period of 18 months. They had all undergone CT imaging and clinical examination by the three gynaecological oncologists who performed the operations (FAS, JD and JK). Their CT images were reviewed in our regular multidisciplinary team meetings (MDT), involving our lead radiologist and all the above operating surgeons. Following this review, only selected patients in whom OCR was deemed possible on CT imaging were then admitted for surgery. All subjects had also been previously examined and were regarded as fit and potentially suitable for up-front surgical intervention. These were therefore regarded as the inclusion criteria. Patients with advanced metastatic stage (stage IV), unfit or in cases OCR was deemed unfeasible, were excluded from the study.

Data gathering and documentation were pro-forma based, recording the operative findings from both minilaparotomy and formal laparotomy. The details of minilaparotomy included the site, length of mini-laparotomy incision, time taken to enter the peritoneal cavity, time taken to finish the procedure and any operative or post operative complication.

Prior to operation, an examination under anaesthesia was performed in all of the cases. A mini-laparotomy incision was made through a midline incision. The incision was sited below, over or above the umbilicus, depending on where the surgeon deemed it most appropriate for full intra- abdominal assessment. The length of the incision was kept to the minimum, allowing only the insertion of one hand after some negotiation. This length usually corresponded to the surgeon's glove size or width of the palm at metacarpophalangeal joint (Fig. 1). To keep the incision length to the minimum, the surgeon placed his hand over the preferred site of incision and marked either side of his knuckles with a marker pen. The length between the two marked points was measured prior to incision and starting time noted.

The palpation findings were charted as main tumour mass, other pelvic and abdominal organs, peritoneal and omental disease. This was followed by visual inspection, usually using a Morris retractor to allow lifting of the anterior abdominal wall. The time taken to enter abdomen, and to complete both palpation and inspection was recorded.

The criteria for OCR were regarded as residual tumour less than $2 \mathrm{~cm}$ in volume. This criteria still remains an acceptable standard in most UK centres. Following inspection and palpation through the mini-laparotomy, the surgeon's impression of achieving OCR was recorded as "possible" (meaning that it may be possible to reduce the volume of disease to less than $2 \mathrm{~cm}$ ), "not possible" or "unsure". The impression was mainly based on palpation findings, as the visual inspection in most cases was limited.

Formal laparotomy was then carried out by the same operator, extending the mini-laparotomy incision, both caudally and cranially. On completion of the surgery, the operating surgeon documented whether "optimal" or "suboptimal" cytoreduction had been achieved.

\section{Findings and analysis}

Data was collected for a total of 50 consecutive patients with suspected stage III/IV ovarian cancer, over a span of 18 months. Data from five cases was regarded as either

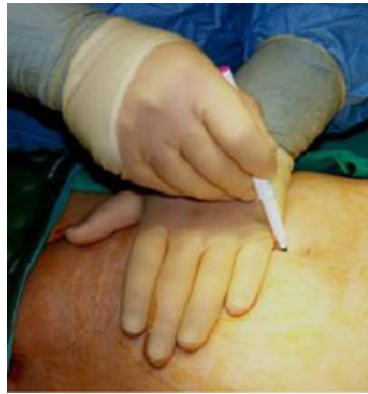

a

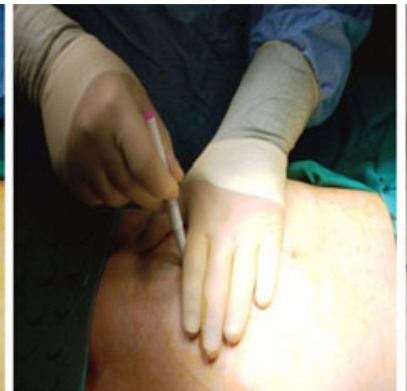

b

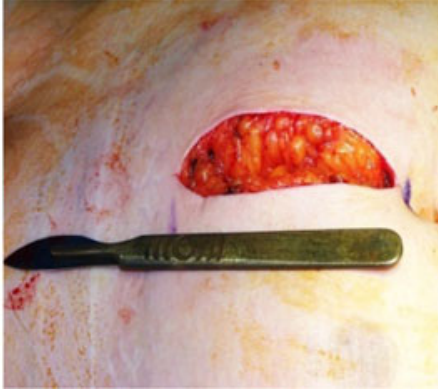

C

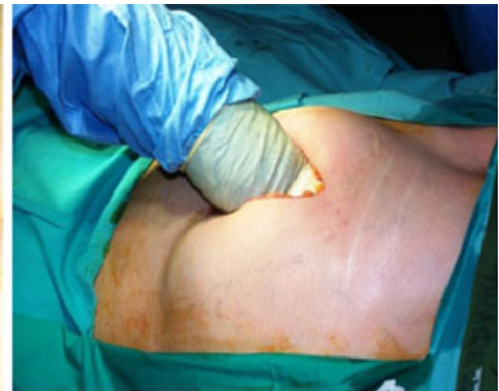

d
Fig. 1 The steps involved in minilaparotomy: demarcation of a suitable midline site and length corresponding to the width of the palm at meta-carpo-phalygeal joint (a and $\mathbf{b}$ ), formation of minilaparotomy was limited to the site marked (c), systemic intraabdominal palpation, examination and assessment of resectability $(\mathbf{d})$ 
inadequate or retrospective and hence excluded. Hence results from 45 cases were analysed. The median age of the patients was 64 years (mean 62.5 years, range $34-80$, SD 11.71). The median BMI was $29.1 \mathrm{~kg} / \mathrm{m}^{2}$ (mean $29.9 \mathrm{~kg} / \mathrm{m}^{2}$, range 18.7-67.5, SD 7.55). The median abdominal girth was $95 \mathrm{~cm}$ (mean $95.87 \mathrm{~cm}$, range 42-148, SD 16.79). Fifteen patients (33\%) had previously undergone abdominal surgery for various gynaecological and non gynaecological reasons. Based on clinical and radiological imaging, stage III disease was suspected in thirty eight (84.44\%) and stage IV in seven cases $(5.56 \%)$. The majority of these patients $(n=40,89 \%)$ underwent upfront surgery prior to adjuvant chemotherapy. Only five patients had received neo-adjuvant chemotherapy $(11 \%)$ prior to their delayed primary surgery.

The median length of the mini-laparotomy incision was $9 \mathrm{~cm}$ (mean $8.59 \mathrm{~cm}$, range 5-12, SD 1.16). The median time to enter the peritoneal cavity, starting from knife to skin, was 4 min (mean 5.10 min, range 1-15, SD 3.04) while the median time taken to complete the palpation and visual exploration was 6.5 min (mean $7.52 \mathrm{~min}$, range 230, SD 4.83).The total median time for making the incision and completion of examinations was $10 \mathrm{~min}$ (with mean of $12.62 \mathrm{~min}$, range 5-45, SD 7.23).

Satisfactory palpation of pelvis and upper abdomen, through the mini-laparotomy incision, was proven to be possible in all cases $(100 \%)$ but visual inspection was limited, particularly for upper abdominal organs.

Following formation of mini-laparotomy, 27 out of the 45 cases were deemed optimally cyto-reducible or "OCR possible". From this "possible" group, OCR was achieved in 24 cases. In eight cases, OCR was regarded as "not possible" and in none of these cases was OCR achieved. In the remaining ten patients, the surgeon was "unsure" and in only three of these cases was OCR achieved (Table 1).

The specificity of mini-laparotomy to predict OCR was noted to be $73 \%$ and sensitivity was $100 \%$. Positive predictive value (PPV) of mini-laparotomy in assessment of OCR was calculated to be $89 \%$ and negative predicative value (NPV) of $100 \%$.

In three cases, OCR was not possible despite initial assessment through mini-laparotomy. In one patient, part of ovarian capsule could not be fully resected away from pelvic side wall. In the second case, failure to achieve OCR was due to tumour plaques in Pouch of Douglas (POD) and over rectum. The third patient had previous total abdominal hysterectomy and residual tumour plaques could not be fully resected from vaginal vault and bladder base.

Out of ten cases commented as "unsure" at minilaparotomy, in only three cases $(30 \%)$ was OCR achieved at laparotomy. Each of these three cases required radical surgery, including bowel resection in two patients.

In eight cases OCR was regarded as not possible after mini-laparotomy examinations and in none of these was OCR achieved after full laparotomy. In four of these patients $(50 \%)$ the main reason was fixed central pelvic mass densely adherent to the adjacent pelvic and abdominal structures. In the other four patients (50\%), extensive upper abdominal disease involving diaphragmatic surfaces, spleen, stomach and porta hepatis were the main reasons precluding OCR. In addition to the above eight cases, there were ten patients that OCR was not achieved ( 3 falsely predicted as "OCR possible" and in seven cases the operator was "unsure"). This was due to following reasons: fixed and densely adherent pelvic mass, extensive supracolic omental mass, significant peritoneal disease and involvement of upper abdominal organs.

There were no complications associated with minilaparotomy. However, excess bleeding according to the surgeon's judgment was reported in three cases $(6 \%)$. The bleeding in all three cases was noted to be from central tumour mass that was adherent to the peritoneum over the site of mini-laparotomy incision.

\section{Discussion}

OCR remains the gold standard treatment for all stages of ovarian cancer. In advanced ovarian malignancy, achievement of OCR has been shown to have a direct impact on patient's disease-free interval and overall survival [9-11]. The definition of OCR has greatly changed over the years: from residual disease of less than $2 \mathrm{~cm}$ to resection or ablation of all macroscopic disease in recent years [12]. However, in most UK centres, a residual disease of less than $2 \mathrm{~cm}$ remains an acceptable goal. Currently the mainstay of preoperative assessment of such patients with regards to resectability and likelihood of achieving OCR are based on clinical examinations, tumour marker levels and CT findings [8-11, 13-16]. It is also common practice in UK to administer neo-adjuvant chemotherapy prior to

Table 1 Comparison of outcome of mini-laparotomy and laparotomy

\begin{tabular}{llll}
\hline & OCR achieved after full laparotomy & OCR not achieved after full laparotomy & Total \\
\hline OCR predicted possible on mini-laparotomy & 24 (true positive) & 3 (false positive) & 27 \\
OCR predicted not possible on mini-laparotomy & 0 (false negative) & 8 (true negative) & 8 \\
Unsure following mini-laparotomy & 3 & 7 & 10 \\
\hline
\end{tabular}


delayed primary surgery in patients that OCR may not appear feasible on initial assessment.

Current preoperative assessments have limited predictability in selection of patients suitable for OCR. The sensitivity of CT in identifying patients that OCR can be achieved is around 60$70 \%$, with varying reported specificity between $70 \%$ and $100 \%$ $[17,18]$. The main drawbacks of CT imaging is the inability to reliably assess the extent of disease in the supra colic compartment, porta hepatis, miliary disease over bowel mesentery and peritoneum, especially in absence of ascites [8-11, 19].

In order to overcome the limitations associated with $\mathrm{CT}$, a few centres routinely perform laparoscopic staging in all their ovarian cancer patients prior to formal laparotomy. This modality of assessment, in experienced hands, has been shown to have PPV of $87 \%$ and NPV of $100 \%$ [20-22]. However, laparoscopic assessment requires an experienced surgical team and laparoscopic resources. In addition, it is associated with increased theatre time and possible increased morbidity. Furthermore, there are reported technical difficulties with the laparoscopic procedure, particularly when intra abdominal adhesions are present in advanced stage disease [23-26]. More importantly, there is a lack of tactile sensation which is of great importance in assessment of resectability and achievement of OCR [27-29]. We believe such shortcomings can be avoided by using a simple minilaparotomy as described in this paper. Furthermore, time required to perform mini-laparotomy would be much less than laparoscopic method due to its simplicity and greater familiarity with the technique. There is no need for a learning curve in mini-laparotomy unlike laparoscopic techniques and is clearly more cost effective.

In our group of patients, all 45 patients were regarded as suitable candidates for OCR following MDT review. However, OCR was achieved in only 27 out of the 45 patients $(60 \%)$. This finding is consistent with reported suboptimal CT accuracy in predicting OCR in the literature [30]. In contrast, following intraabdominal palpation through mini-laparotomy, the accuracy for predicting OCR was much higher at $89 \%$ (24 of 27 cases). More importantly, OCR was not achieved in any of the eight patients where it was predicted that OCR would not be possible at mini-laparotomy, reflecting an NPV of $100 \%$. This has great clinical importance in avoiding unnecessary major laparotomy. In addition, considering small size and lack of morbidity associated with mini-laparotomy, it should result in much quicker recovery and hence shorten delay in receiving neo-adjuvant chemotherapy.

We acknowledge the principle shortcoming of the study to be the small sample size, hence potential for type II statistical error. However, despite this limitation, we believe the findings to be of clinical importance.
In mini-laparotomy, one could encounter a degree of visual and tactile restriction as compared with abdominal and pelvic assessment following full laparotomy. This may explain the reason why in nearly $20 \%$ of cases the operating surgeon was unsure of ability to obtain OCR. However, it is of interest to note that in this group of patients OCR was only achieved in $30 \%$. One therefore could conclude that mini-laparotomy may also help surgeon in making a balanced decision, as to proceed or not in the "unsure" group of patients.

In order to overcome the visual limitation of minilaparotomy, one may consider a combination of the techniques of laparoscopy and mini-laparotomy, better known as hand-assisted laparoscopy. However such technique will be inevitably associated with increased theatre time and operative complexity. In future, it is anticipated that integrated FDG-PET/CT may have a role in assessment of disease spread and decision with regards to best therapeutic strategy in ovarian cancer. FDG-PET/CT has been shown to have a higher sensitivity (91\%) than PET, CT or MRI in assessment of disease spread [31,32]. Hence, it may further assist in early identification of patients who are not suitable for up front surgery.

\section{Conclusions}

Despite our small sample size, this study has shown minilaparotomy to be a safe, simple and effective technique in assessment of tumour resectability and chances of obtaining OCR. More importantly it is very accurate in identifying patients unsuitable for primary cytoreduction. By avoiding an unnecessary full laparotomy, patients' recovery will be expedited and chemotherapy could be initiated earlier.

Conflicts of interest The authors report no conflicts of interest. The authors alone are responsible for the content and writing of the paper.

\section{References}

1. Information Services Division, NHS National Services Scotland: Cancer in Scotland. Sept 2008, page 4

2. Junor EJ, Hole DJ, McNulty L, Mason M, Young J (1999) Specialist gynaecologists and survival outcome in ovarian cancer: a Scottish national study of 1866 patients. Br J Obstet Gynaecol 106:1130-1136

3. Kristensen GB, Trope C (1997) Epithelial ovarian carcinoma. Lancet 349:113-117

4. Information Services Division, NHS National Services Scotland: Cancer in Scotland. Sept 2008, p 13

5. Hunter RW, Alexander NDE, Soutter WP (1992) Meta-analysis of surgery in advanced ovarian carcinoma: is maximum cytoreductive surgery an independent determinant of prognosis? Am J Obstet Gynecol 166:504-511 
6. Hoskins WJ, Bundy BN, Thigpen JT et al (1992) The influence of cytoreductive surgery on recurrence-free interval and survival in small-volume stage III epithelial ovarian cancer: a Gynecologic Oncology Group study. Gynecol Oncol 47:159-166

7. Allen DG, Heintz AP, Touw FW (1995) A meta-analysis of residual disease and survival in stage III and IV carcinoma of the ovary. Eur J Gynaecol Oncol 16(5):349-356

8. Hacker NF, Berek JS, Lagasse LD, Nieberg RK, Elashoff RM (1983) Primary cytoreductive surgery for epithelial ovarian cancer. Obstet Gynecol 61:413-420

9. Hogberg T (1995) Primary surgery in ovarian cancer: current opinions. Ann Med 27:95-100

10. Voest EE, van Houwelingen JC, Neijt JP (1989) A meta-analysis of prognostic factors in advanced ovarian cancer with median survival and overall survival (measured with the log [relative risk]) as main objectives. Eur J Cancer Clin Oncol 25:711-720

11. Chen SS, Bochner R (1985) Assessment of morbidity and mortality in primary cytoreductive surgery for advanced ovarian carcinoma. Gynecol Oncol 20:190-195

12. Winter WE, Maxwell GL, Tian C et al (2007) Prognostic factors for stage III epithelial ovarian cancer: a Gynecologic Oncology Group Study. J Clin Oncol 25:3621-3627

13. Bristow RE, Duska LR, Lambrou NC, Fishman EK, O'Neill MJ, Trimble EL, Montz FJ (2000) A model for predicting surgical outcome in patients with advanced ovarian carcinoma using computed tomography. Cancer 89:1532-1540

14. Nelson B, Rosenfeld AT, Schwartz PE (1993) Preoperative abdominopelvic computed tomographic prediction of optimal cytoreduction in epithelial ovarian carcinoma. J Clin Oncol $11: 166-172$

15. Forstner R, Hricak H, Occhipinti KA, Powell CB, Frankel SD, Stern JL (1995) Ovarian cancer: staging with CT and MR imaging. Radiology 197:619-626

16. Sanders RC, McNeil BJ, Finberg HJ et al (1983) A prospective study of computed tomography and ultrasound in the detection and staging of pelvic masses. Radiology 146:439-442

17. Kurtz AB, Tsimikas JV, Tempany CM et al (1999) Diagnosis and staging of ovarian cancer: comparative values of Doppler and conventional US, CT, and MR imaging correlated with surgery and histopathologic analysis - report of the Radiology Diagnostic Oncology Group. Radiology 212:19-27

18. Bristow RE (2006) Predicting unresectable ovarian cancer: taking aim at a moving target. Editorial. Gyn Oncol 100(3):449-450

19. Kubik-Huch RA, Dörffler W, von Schulthess GK, Marincek B, Köchli OR, Seifert B, Haller U, Steinert HC (2000) Value of (18 F)FDG positron emission tomography, computed tomography, and magnetic resonance imaging in diagnosing primary and recurrent ovarian carcinoma. Eur Radiol 10:761-767
20. Funt SA, Hricak H, Abu-Rustum N, Mazumdar M, Felderman H, Chi DS (2004) Role of CT in the management of recurrent ovarian cancer. AJR Am J Roentgenol 182:393-398

21. Axtell AE, Lee MH, Bristow RE et al (2007) Multi-institutional reciprocal validation study of computed tomography predictors of suboptimal primary cytoreduction in patients with advanced ovarian cancer. J Clin Oncol 25:384-389

22. Dowdy S, Mullany S, Brandt K, Huppert B, Cliby W (2004) The utility of computed tomography scans in predicting suboptimal cytoreductive surgery in women with advanced ovarian carcinoma. Cancer 101:346-352

23. Meyer JI, Kennedy AW, Friedman R (1995) Ovarian carcinoma: value of $\mathrm{CT}$ in predicting success of debulking surgery. Am J Roentgenol 165:875-878

24. Deffieux X, Castaigne D, Pomel C (2006) Role of laparoscopy to evaluate candidates for complete cytoreduction in advanced stages of epithelial ovarian cancer. Int J Gynecol Cancer 16:35-40

25. Angioli R, Palaia I, Zullo MA, Muzii L, Manci N, Calcagno M, Panici PB (2006) Diagnostic open laparoscopy in the management of advanced ovarian cancer. Gynecol Oncol 100:455-461

26. Fagotti A, Fanfani F, Ludovisi M, Lo Voi R, Bifulco G, Testa AC, Scambia G (2005) Role of laparoscopy to assess the chance of optimal cytoreductive surgery in advanced ovarian cancer patients: a pilot study. Gynecol Oncol 96:729-735

27. Fagotti A, Ferrandina G, Longo R, Mancuso S, Scambia G (2002) Minilaparotomy in early endometrial cancer: an alternative to standard and laparoscopic treatment. Gynecol Oncol 86:177-183

28. Nicoletto MO, Tumolo S, Talamini R et al (1997) Surgical second look in ovarian cancer: a randomized study in patients with laparoscopic complete remission - a Northeastern Oncology Cooperative Group-Ovarian Cancer Cooperative Group Study. J Clin Oncol 15:994-999

29. Abu-Rustum NR, Barakat RR, Siegel PL, Venkatraman E, Curtin JP, Hoskins WJ (1996) Second-look operation for epithelial ovarian cancer: laparoscopy or laparotomy? Obstet Gynecol $88: 549-553$

30. Clough KB, Ladonne JM, Nos C, Renolleau C, Validire P, Durand JC (1999) Second look for ovarian cancer: laparoscopy or laparotomy? Prospective comparative study. Gynecol Oncol 72:411-417

31. Risum S, Høgdall C, Loft A, Berthelsen AK, Høgdall E, Nedergaard L, Lundavall L, Engelholm SA (2008) Prediction of suboptimal primary cytoreduction in primary ovarian cancer with combined positron emission tomography/computed tomography-a prospective study. Gyn Oncol 108:265-270

32. Kitajima K, Murakami K, Sakamoto S, Kaji Y, Sugimura K (2011) Present and future of FDG-PET/CT in ovarian cancer. Ann Nucl Med 25:155-164 